\title{
Carpal tunnel syndrome diagnosis: validation of a clinic-based nerve conduction measurement device
} \author{
Uolevi Tolonen ${ }^{2}$

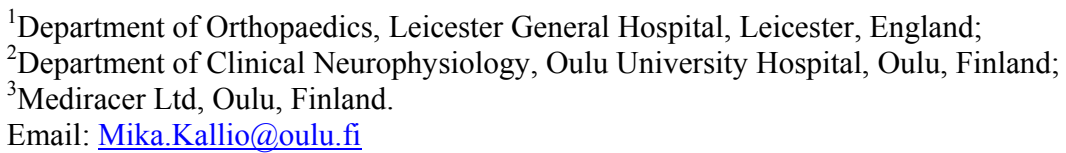

Timothy P. Green ${ }^{1}$, Mika Kallio ${ }^{2}$, Malcolm R. A. Clarke ${ }^{1}$, Pankaj Pathak ${ }^{1}$, Veijo Lesonen ${ }^{3}$,

Received 1 February 2011; revised 3 March 2011; accepted 8 March 2011.

\begin{abstract}
Background: Carpal Tunnel Syndrome (CTS) is the commonest upper limb nerve entrapment syndrome seen in practice. In many centres, nerve conduction studies (NCS) have been adopted as a routine part of the diagnostic process. In the United Kingdom, the time taken to access diagnostic tests has been likened to a "hidden waiting list", lengthening the time taken for a patient to access treatment. In the current healthcare climate with a centrally driven aim to reduce patient waiting time to a maximum of eighteen weeks, including tests, such waiting is even more unacceptable. Aim. This study was performed in order to evaluate a simple handheld device for quantifying median nerve lesions in CTS. Design of study: A prospective blinded cohort study. Setting: Leicester General Hospital, Carpal Tunnel Service Method: Participants were recruited from the normal referral stream. If the clinical findings were consistent with a diagnosis of CTS, they were formally consented to the study in which results from the new handheld device were compared with traditional NCS. Final test group consisted of 63 participants. Results: For the new device the correct positive detection rate for abnormal nerve conduction was $91 \%$ ( $74 / 81$ hands). Of the seven abnormal results not picked up by the new device, four were in asymptomatic hands (positive per cent agreement in symptomatic hands $95 \%$ ). There were no false positives with the new system. (Negative per cent agreement $100 \%$ ) Conclusion: We conclude that this new device demonstrates a high degree of concordance with currently available traditional NCS. The study suggested ways in which the accuracy could be further improved.
\end{abstract}

Keywords: Carpal Tunnel Syndrome; Nerve Conduction Studies; Diagnosis; Portable

\section{INTRODUCTION}

Carpal Tunnel Syndrome (CTS) is the most common upper limb nerve entrapment syndrome [1,2]. Diagnosis is largely based on symptoms and clinical signs, but these are of variable sensitivity and specificity. In many centres, nerve conduction studies (NCS) have been adopted as a routine part of the diagnostic process despite evidence that up to $16 \%$ of patients may have normal studies in the presence of clinically proven CTS $[3,4]$. Insisting on NCS for every patient satisfies the clinicians' need for objective evidence but adds a significant extra expense and delay to the diagnosis and treatment of this very common condition. It may also introduce a false negative, failing to diagnose CTS when it is present [1].

In the United Kingdom, the time taken to access diagnostic tests is several weeks. With the current centrally driven aim to reduce patient treatment time, including tests, to a maximum of eighteen weeks, delays of months are unacceptable.

A new, portable device that can simply and cheaply diagnose a median nerve lesion and quantify its severity in the clinic may have a role in establishing the diagnosis. In conjunction with clinical findings this could reduce both treatment times and the number of hospital visits. A good practice guide produced by the Department of Health [5] suggests that this device is "worthy of further assessment".

This paper describes a study designed to compare results from the new device with traditional NCS.

\subsection{Background}

Our Carpal Tunnel Service was set up in 1999 in order 
to improve the service we provided to those affected by this condition in our local community. It has proved to be very successful, reducing overall treatment time from 106 weeks to 6 weeks. Over six and a half thousand people have been treated to date. The achievements of the service have been presented at local and national meetings and have attracted recognition from the Department of Health.

In Oulu, Finland, a group of Clinical Neurophysiologists developed a hand-held device for diagnosing the median nerve lesion in CTS based on the work of Uncini et al. [6] A clinical trial to prove its effectiveness was performed [7], but was slow to recruit patients in a country with a population of only five million people. Finpro, an agency set up to support Finnish innovation contacted the Department of Health in the UK to locate clinical partners that could help validate the system further. This study is the result of a collaborative work between our unit and Oulu.

\section{METHODS}

The study was based in secondary care in a single hospital that is the normal base for the Carpal Tunnel Service. Approval was sought from and granted by the local Ethics Committee.

The study set out to compare results from the new device with traditional nerve conduction studies. The new device is a portable, hand-held instrument designed to be used by non-specialists and so was operated by local clinicians after a brief familiarisation period. Experienced clinical neurophysiologists from Finland carried out the traditional nerve conduction studies for comparison.

The null hypothesis was that there would be no significant difference between the two systems.

In order to overcome the practicalities in getting the two research teams together, this prospective cohort study was set up to be completed over three days. Concentrating the measurements into a short time avoided some of the variables in testing, such as ambient temperature.

All participants had both hands tested by each method regardless of whether or not both hands were symptomatic. During testing, the British and Finnish groups were blinded to the results obtained by the other group. The study was submitted to the local ethics committee (University Hospitals of Leicester) January 2006 and passed by them on first review.

\subsection{Participants}

Participants were recruited from the normal referral stream to our Carpal Tunnel Service. They were referred by their General Practitioners or from other Specialists within secondary care. The referring doctors all thought their patients exhibited symptoms suggestive of carpal tunnel syndrome. (i.e. tingling and numbness affecting the radial side of the hand). There were no other exclusion criteria.

All potential participants $(n=120)$ were sent a letter inviting them to take part in the study. All those that accepted $(n=104)$ were clinically assessed. A symptom form was completed which included the distribution of any sensory disturbance. If the clinical findings were consistent with a diagnosis of CTS, they were formally recruited to the study, consented and allocated onto one of the three study days. Any participant whose clinical assessment suggested an alternative diagnosis, a more comprehensive problem than a mere suspicion of CTS, or who declined to continue in the study was referred on as appropriate or transferred into the standard Carpal Tunnel Service.

The final number of participants recruited into the study was 65 of whom 49 were female and 16 were male. The age range was from 23 to 83 (mean 52.3) years. Two of the recruited cohort had to cancel on the day of their study because of personal reasons, leaving a test group of 63 (48 females, 15 males, aged 23 - 81, mean 52.8) equating to a total of 126 tested hands. Clinical symptoms of CTS were unilateral in 34 participants and bilateral in 29.

\subsection{Interventions}

Each participant had base line measurements of weight and height recorded before being allocated to one of the Clinical Neurophysiologists so that a traditional set of neurophysiological measurements could be carried out. Orthodromic sensory conduction latencies were measured by stimulating index and ring fingers with felt pad electrodes and recording at wrist $1 \mathrm{~cm}$ above the distal wrist groove. This is the method used also by the new device. Additionally, the traditional measurement included mixed conduction velocities of the median and ulnar palm to wrist segment of $8 \mathrm{~cm}$. Finally, median and ulnar distal motor latencies were examined with $7 \mathrm{~cm}$ interelectrode distance. (Measurements were made using Keypoint ${ }^{\circledR} 4$ and Keypoint ${ }^{\circledR}$ Portable, Medtronic, Skovlunde, Denmark). The participants were then randomly allocated to one of the local clinicians so as to have nerve conduction studies carried out using the new device. (Mediracer ${ }^{\circledR}$, Mediracer International, Oulu, Finland). The new device uses two self-adhesive, disposable hydrogel coated electrodes. One is a stimulating electrode that wraps around the proximal phalange of the finger being tested and the second is a recording electrode that is placed over the wrist $1 \mathrm{~cm}$ above the distal wrist groove (Figure 1). 


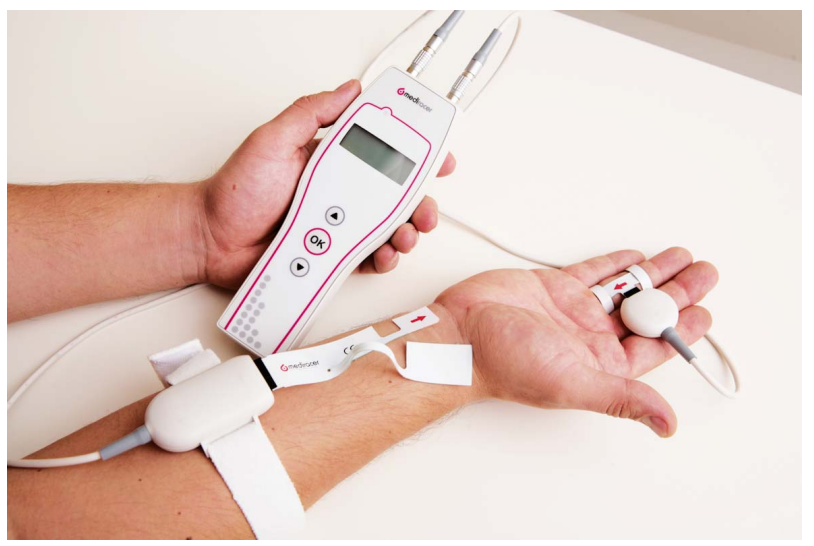

Figure 1. The new device (Mediracer ${ }^{\circledR}$, Mediracer International, Oulu, Finland) uses two self-adhesive, disposable hydrogel coated electrodes. One is a stimulating electrode that wraps around the finger being tested and the second is a recording electrode that is placed over the wrist.

Tests are made on the ring finger which is innervated by both median and ulnar nerves plus the index finger where there is only median nerve innervation. The handpiece is attached to the electrodes and has an automatic menu for the operator. The test begins once background muscle activity drops below threshold.

A stimulus is gradually increased until the participant becomes just aware of it. The stimulus is then automatically increased by $250 \%$ and repeated sixty-four times over 35 seconds. Overall test time is in the order of five minutes. The handset shows how many stimuli were successfully received, allowing the test to be repeated if necessary. Data is downloaded by a Bluetooth ${ }^{\mathrm{TM}}$ link to a computer, where the software calculates and displays a nerve conduction latency/amplitude graph (Figure 2).

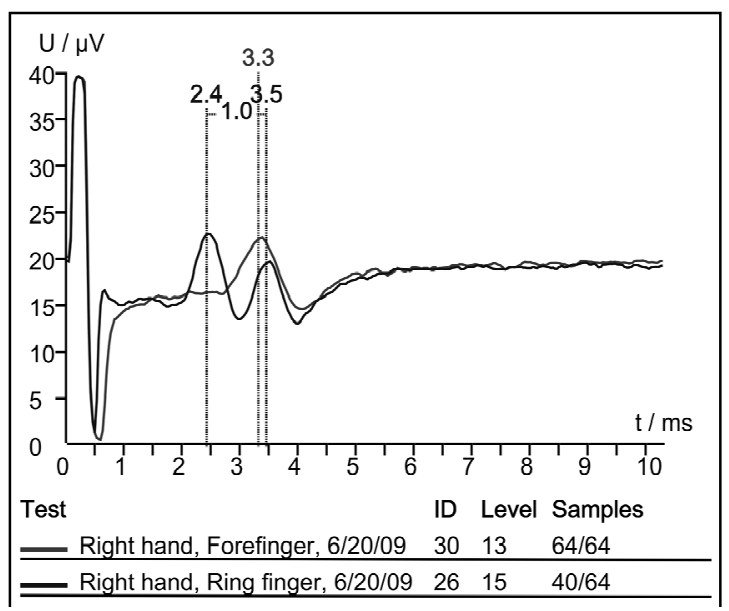

Figure 2. Double peak shown after ring finger stimulation (blue line). Interpeak distance $1.0 \mathrm{~ms}$ is consistent with mild median nerve damage (peak latencies rounded). Forefinger response (red line) is also significantly longer $(0.9 \mathrm{~ms})$ when compared to the first peak from ring finger.
All the graphs were visually checked off line and peak latency cursors reset if needed by a specialist in clinical neurophysiology who was not aware of any other patient data. The peak latencies needed adjustments in 13 patients.

Following the two different NCS tests, the participants were seen by one of two other local clinicians to review the results of their tests and decide their further management.

\subsection{Main Outcome Measures}

Each participant was assessed to determine whether their symptoms were suggestive of CTS. The presence of symptoms in either hand was recorded, as was any previous surgery. Hand dominance and occupation were noted. The Boston Hand Score [8] for CTS was used to quantify symptom severity (SSS) and functional severity (FSS).

The neurological severity of CTS was classified in a simplified manner. In the present study neurophysiological severity classification was obtained from traditional measurements by using Padua et al.'s [4] five abnormal NCS classes which have been reduced to threemild (includes minimal), moderate and severe (includes extreme). The reasons for this are discussed later. In our series there were nine minimal lesions included to the mild class and one extreme case included to the severe group. In the new device study the same three severity classes were scaled using reference values obtained from the earlier study [7]. These were determined fitting the new device data to the Padua et al.'s [4] scales of the traditional device measurements. For the new device, mild and moderate severity can be assessed using only measurements from the ring finger, as separate responses from median and ulnar nerves are easily distinguished as seen in Figure 2. In severe cases however, no median nerve signal may be seen. In this case, adding the result from stimulation of the forefinger will confirm the absence of a median signal and avoid missing a severe case. (Table 1) The absolute values for the new device study latencies are taken from the previous study [7].

In order to test the agreement of the similarly measured nerve responses between the Mediracer and Keypoint devices, scatter plots with linear regression line fit and Bland-Altman plots [9] for the latency differences between ring finger median (4PM) and ulnar nerve (4PU) responses and ring finger (4PU) and index finger (2P) responses were calculated. This was analysed using $\mathrm{R}$ statistics and OriginPro 8 software. Pearson's and intraclass correlation coefficients were also calculated using the SPSS 17.0 software.

\section{RESULTS}

There were 92 symptomatic hands in 63 participants, of 
Table 1. Reference values for latency differences using the new device.

\begin{tabular}{|c|c|c|c|}
\hline \multicolumn{4}{|c|}{ Latency differences between ring finger peaks } \\
\hline Normal & Mild & Moderate & Severe \\
\hline$\leq 0.7 \mathrm{~ms}$ & $0.8-1.1 \mathrm{~ms}$ & $\geq 1.2 \mathrm{~ms}$ & $\begin{array}{l}\text { A single ulnar } \\
\text { derived peak. } \\
\text { No apparent } \\
\text { median response }\end{array}$ \\
\hline
\end{tabular}

Latency differences between ring and forefinger peaks

\begin{tabular}{|c|c|c|c|}
\hline Normal & Mild & Moderate & Severe \\
\hline$\leq 0.5 \mathrm{~ms}$ & $0.6-0.9 \mathrm{~ms}$ & $\geq 1.0 \mathrm{~ms}$ & $\begin{array}{l}\text { Confirmation of } \\
\text { no median peak } \\
\text { from forefinger }\end{array}$ \\
\hline
\end{tabular}

Limits for carpal tunnel severity classes using the new device. If both fingers are stimulated, as they were in this study, it will be seen that different classifications might be arrived at for the same hand. In such a case the more severe classification was accepted.

whom 21 were right, 13 were left and 29 were bilateral. Ten participants had had previous carpal tunnel surgery on one hand up to sixteen years before, but none of these operated hands were still symptomatic.

For symptomatic hands the Boston Hand Score showed a mean symptom severity score of 32.6 and a functional severity score of 19.0 (Normal or asymptomatic scores are 11 SSS and 8 FSS)

The neurophysiological testing produced a table of results for both testing systems (Table 2).

There were seven hands where differences were seen between the two systems. Four of these seven were in asymptomatic hands. (Nos. 7, 10, 17 and 22). Two of these had previously undergone carpal tunnel decompressions. Traditional NCS picked up mild residual

Table 2. The severity of CTS in clinically symptomatic and non-symptomatic hands using traditional and new device nerve conduction velocity measurements.

\begin{tabular}{cccc}
\hline & & Traditional NCS & New Device \\
\hline & Normal & 31 & 33 \\
Symptomatic & Abnormal & 61 & 58 \\
hands (92) & Mild & 9 & 12 \\
& Moderate & 43 & 31 \\
& Severe & 9 & 15 \\
& Non-diagnostic & 0 & 1 \\
\hline \multirow{3}{*}{ Non-symptomatic } & Normal & 14 & 18 \\
hands (34) & Abnormal & 20 & 16 \\
& Mild & 11 & 11 \\
& Moderate & 8 & 4 \\
\hline Totals & Severe & 1 & 1 \\
\hline
\end{tabular}

sensory nerve conduction abnormalities in all four and a mild motor nerve conduction abnormality in one. In comparison, the new device recorded these asymptomatic hands as normal.

These differences using the new device may be explained by a dilution effect from the longer distance the finger stimulation method uses. In traditional NCS the nerve is measured in addition to finger-to-wrist segment over the palm-to-wrist segment. This is also the reason we used a different classification in this study.

Non-significant mild deficits could be harder to detect with the new device using only finger-to-wrist segment. Another, albeit rare, explanation is the presence of a communicating branch from the median side of the ring finger in the palm to the ulnar nerve. Finally, low stimulus intensity (2.5 times sensory threshold) is not always supramaximal and might not evoke a response in all axons. The remaining three hands were symptomatic (Nos. 35,47 and 50 ). They had mean symptom severity scores of 32.3 and mean functional severity scores of 20.0. This is not statistically significant from the rest of the symptomatic group. Nos. 35 and 50 had however, only very mild sensory conduction abnormalities. No. 35 also had a mild motor conduction abnormality. Both cases were negative using the new device and again, the reasons suggested above may explain the different results. The final case (No. 47) had a latency difference classified as severe on traditional testing whereas there was no response in either ring finger stimulation or forefinger stimulation using the new device. Missing median and ulnar nerve responses may be due to polyneuropathy or because of simultaneous median and ulnar nerve lesion and these patients should always be re-directed to traditional NCS.

When testing only symptomatic hands that were abnormal on traditional NCS, the new device correctly found abnormalities in 58 out of 61 hands (positive percent agreement $95 \%$ ). No hands showed a false positive result with the new device (negative per cent agreement $100 \%$ ). In this study, the new device classified the severity of CTS of most of the patients into the same classes as the traditional NCS (Table 2). There was a trend in that the new device recorded more severe cases than the traditional instrumentation. This was probably due to the stimulus intensity level used in this series, i.e. two and a half times a participant's sensory threshold. This will not always be supra maximal, i.e. does not necessarily stimulate all nerve fibres. This could be overcome by choosing higher initial stimulus intensities.

Figures 3(a)-(c) present the agreement of ring and fore finger latency differences (4PM-4PU and 2P-4PU) between the two systems. In Figure 3(b), there is one outlier on the ring finger 4PM-4PU latency differences, 


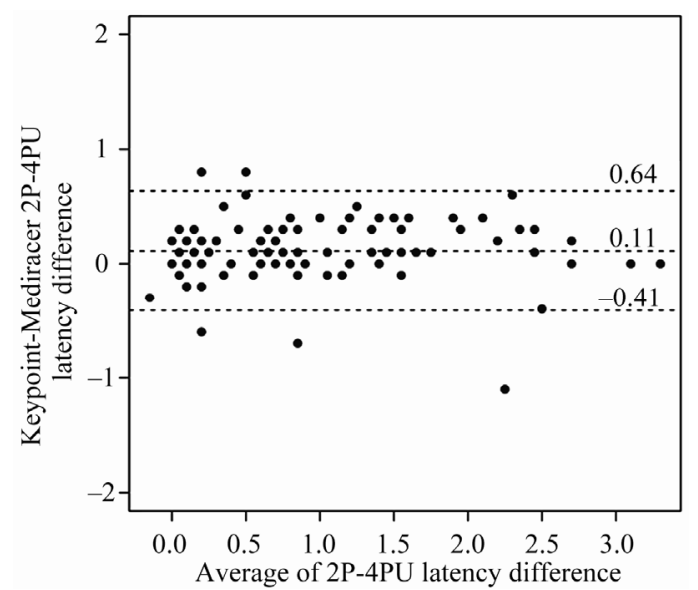

(a)

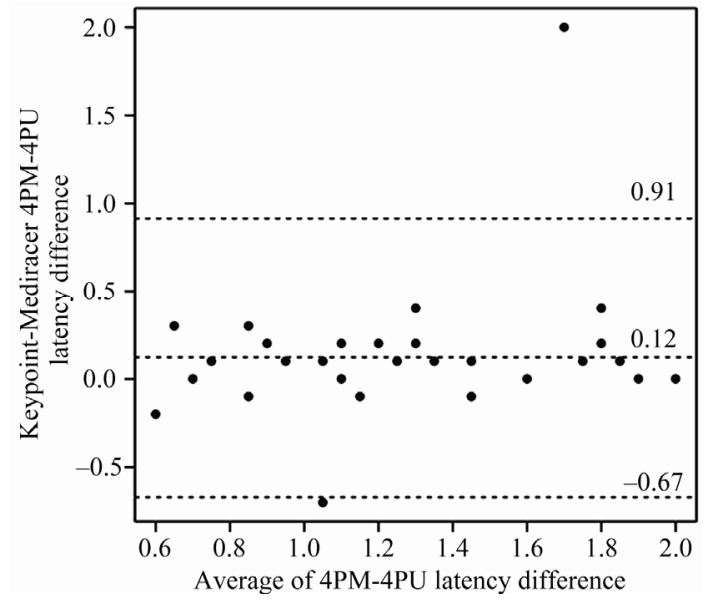

(b)

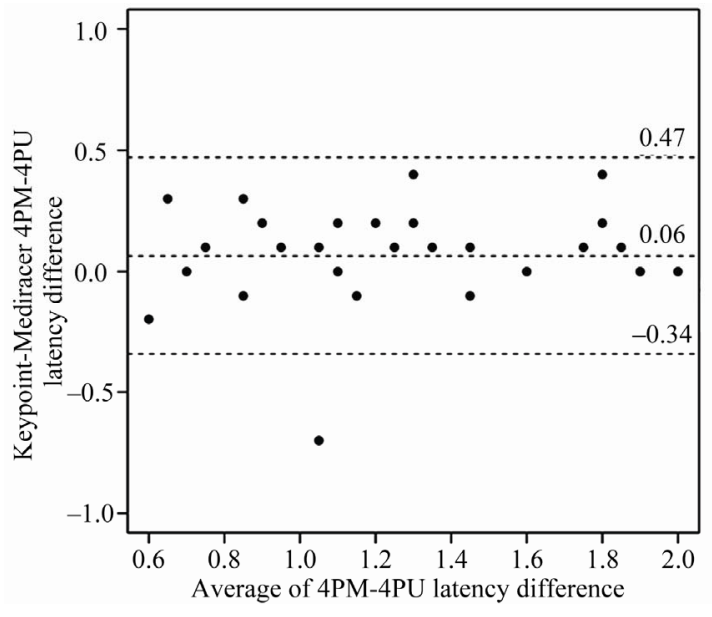

(c)

Figures 3. The graphs displays agreement levels between Keypoint and Mediracer fore and ring finger latency differences. (a) Difference between fore (2P) and ring finger (4PU) latencies between Keypoint and Mediracer. (b) Difference between ring finger double peak (4PM-4PU) latencies showing one outlier and with outlier removed in. (c) Horizontal lines showing values are drawn at the mean difference, and at the upper and lower limits of agreement. that shifts the upper agreement to a non-acceptable level. In a 79 year old lady (No. 4) standard nerve conduction measurements gave a double peak with $2.7 \mathrm{~ms}$ interpeak interval and Mediracer measurement gave $0.7 \mathrm{~ms}$ interpeak conduction time. If this outlier is removed the agreement levels are very acceptable as shown in Figure 3(c).

Figure 4 presents scatter plot of 4PM-4PU latency differences in the two systems and linear regression fit with and without the outlier, showing no important systematic difference. It is possible that this outlier was due to an error in the traditional NCS measurement. When using a felt pad stimulating electrode and rather low stimulus intensity it is possible to evoke cathode stimulation on the ulnar side and anode stimulation on median side of the ring finger. This possibility is supported by the fact that in index finger stimulation both the traditional and the new system produced similar latencies (3.1 vs. $3.3 \mathrm{~ms}$ ).

Pearson's and intraclass correlation coefficients are presented in Table 3. There was high correlation between the devices in the ring finger double peak and ring and forefinger peak latency differences. For the absolute latencies the correlation was not good as the new device missed some median nerve responses that traditional device did not. However, in spite of that latency discrepancy, these few cases were neurophysiologically classified as clearly abnormal in both methods: in the traditional measurement moderately abnormal and in the new device study severely abnormal.

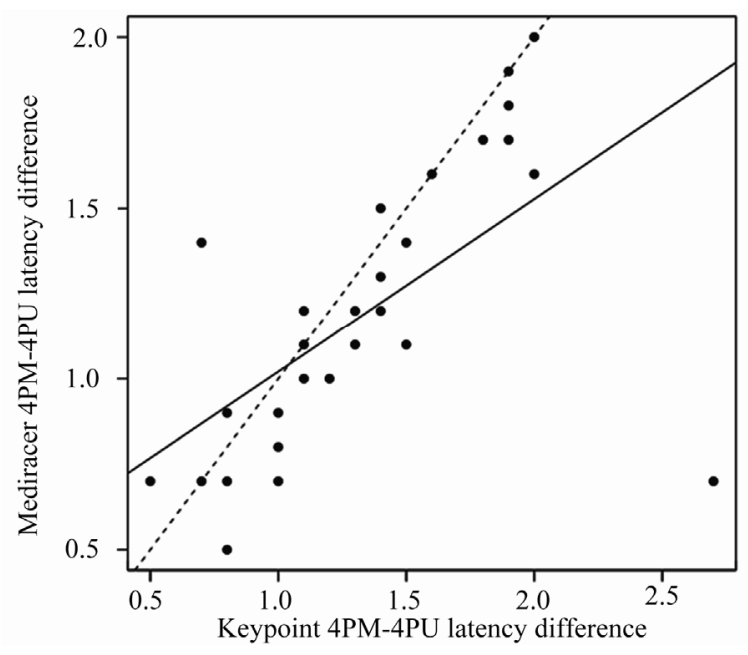

Figure 4. Scatterplot of 4PM-4PU latency differences in the two systems and linear regression fit with (solid line) and without the outlier (dashed line). The estimate slope is 0.54 , intercept 0.49 and respective $95 \%$ confidence intervals are $(0.42,0.66)$ and $(0.34,0.63)$. Without the outlier regression slope and intercept estimates are 0.77 and 0.25 , respective $95 \%$ confidence intervals $(0.68,0.87)$ and $(0.13,0.36)$. 
Table 3. Interexaminer summary statistics and reliability results for mediracer and keypoint nerve conduction studies.

\begin{tabular}{|c|c|c|c|c|c|c|c|}
\hline & \multicolumn{2}{|c|}{ Traditional device } & \multicolumn{2}{|c|}{ New device } & \multirow{2}{*}{$\begin{array}{l}\text { Pearson } \\
\text { correlation }\end{array}$} & \multirow{2}{*}{$\begin{array}{c}\text { Paired t-test } \\
\text { (P-value) }\end{array}$} & \multirow{2}{*}{$\begin{array}{l}\text { Intraclass } \\
\text { correlation }\end{array}$} \\
\hline & Mean (SD) & Range & Mean (SD) & Range & & & \\
\hline \multicolumn{8}{|c|}{ Ring finger stimulation } \\
\hline 4PU latency & $2.5(0.28)$ & $2.0-3.1$ & $2.6(0.43)$ & NR - 3.3 & 0.20 & 0.019 & 0.26 \\
\hline 4PM latency & $4.2(1.1)$ & NR -8.0 & $3.9(0.8)$ & NR - 5.9 & 0.93 & 0.201 & 0.96 \\
\hline 4PM-U latency & $1.7(1.1)$ & $0.6-5.8$ & $1.3(0.7)$ & $0.2-2.9$ & 0.96 & 0.016 & 0.97 \\
\hline \multicolumn{8}{|c|}{ Forefinger stimulation } \\
\hline 2P latency & $3.8(0.99)$ & $2.5-5.7$ & $2.9(0.22)$ & NR - 5.7 & 0.27 & 0.004 & 0.29 \\
\hline 2P-4PU latency & $1.3(1.02)$ & $-0.2-3.5$ & $1.1(0.89)$ & $-0.2-3.3$ & 0.96 & 0.004 & 0.98 \\
\hline
\end{tabular}

$\mathrm{NR}=$ No response, latencies in $\mathrm{ms}$.

\section{DISCUSSION}

The number of patients being referred with a diagnosis of carpal tunnel syndrome is rising in the United Kingdom and abroad $[1,2,10]$. It accounts for almost $10 \%$ of all referrals to our elective Orthopaedic unit.

There seems little doubt that surgical treatment is effective, but there is still debate as to which patients need surgery. A tendency towards milder cases presenting earlier makes clinical diagnosis less reliable $[2,10]$. Any attempt to monitor a clinically mild presentation of CTS by repeated tests has normally been precluded by the difficulty and expense of accessing such tests. Even single diagnostic NCS examinations may be subject to long delays.

Clinical protocols have been developed to reduce the need for NCS. This is a pragmatic solution but may not represent the best or most accurate assessment of the patient who presents with symptoms suggestive of CTS. Introducing a new pathway in which the confidence engendered by NCS can be added to clinical protocols, while speeding and simplifying the process would be an ideal solution. Moreover, since up to $25 \%$ of patients present at a sufficiently late stage such that their chances of successful treatment have already been prejudiced, early diagnosis is important [2].

Where clinical signs are positive but NCS are negative, it could be argued that easy access to repeat testing is imperative if a decision not to operate is made. Not all the parameters of standard NCS are required or even understood by many clinicians. Alterations of nerve conduction speeds can be subtle and affected by many conditions, but in carpal tunnel syndrome with good clinical signs, a simple yes/no answer as to whether there is a sensory median nerve conduction delay between finger and wrist may act as useful confirmation. Quantification of the severity of such a conduction delay can help guide treatment options. Pure motor involvement of the median nerve is rare $-0.3 \%$ $-1 \%[7,11]$.

The new device under test uses peak latency difference between the median and ulnar nerves as the main measurement parameter. This was chosen after an earlier observation showed that latency difference is more sensitive than SCV in the diagnosis of median nerve lesion in CTS [12]. Moreover, the measurement of amplitudes seems not to increase the number of abnormalities detected by using only latency measurement [13]. For this reason and for making the method as simple as possible to use, the standard anatomical landmarks and not the standard distances were chosen for use in the new device NCS.

In $9 \%$ of all hands, in $5 \%$ of symptomatic hands, the new device missed the abnormality detected by the traditional examination. Six of these seven hands showed a mild or very mild abnormality and one a severe abnormality. In this last case the new device did not find any responses either in median nerve or ulnar nerve measurements and a pure median nerve lesion diagnosis could not be made. This loss of both signals is easy to recognize and suggests referral for traditional NCS. CTS with a mild or absent median nerve lesion is usually treated conservatively, and so false negative results will not lead to incorrect treatment. In the severity classification of CTS the new device slightly over exaggerated severe cases. This was possibly due to submaximal stimulation intensities in some measurements.

The small number of incorrect results from the new device study suggested some ways to improve it. The risk of using too weak a stimulus current has been corrected in the latest version. The responses are now always visually checked in real time on the computer screen during the stimulation process in order to ensure supramaximal stimulus. As well as that, in order to confirm the ring finger ulnar nerve response measured in this study, the little finger response should also always be measured. The two digital nerves in this finger give a greater axonal volume and thus a more accurate and sensitive ulnar response compared with that in the ring finger. This way, possibly more low responses could be recorded by the new device, reducing the number of the lost responses and thereby also the slight 
over exaggeration of severe lesions in the present study.

Comparison of the two methods of NCS testing shows a high degree of agreement between the systems. When coupled with clinical tests and a symptom and functional severity score, the diagnosis and quantification of severity of CTS can be supported with a high degree of confidence using the new device However, when only sensory nerve conduction (SNC) measurements are made, and a limited number of nerves are studied, the present new method should only apply to patients with a CTS as in the present study or in a high suspicion of that. In these cases the probable median nerve lesion and its severity can be defined, supplementing the clinical diagnosis of CTS and thus helping the choice of conservative or operative therapy. In cases with clinical diagnostic dilemma, traditional neurophysiology should be primarily consulted.

The present study showed that automatic cursor placements of the new device study missed in several cases. For this reason the cursor setting must always be checked visually as would also happen in traditional NCS. When using the new device in primary or non-specialist practice the interpretation of the data can be obtained via the internet from a specialist in clinical neurophysiology and this would be our recommendation.

The simplicity of the hand-held system and the possibility of it being used by a non-specialist suggest the definite possibility of having a "one-stop" clinic for the validated diagnosis of the condition of carpal tunnel syndrome. The ability to refer results to a specialist in clinical neurophysiology via a web-based system for a report increases the usefulness of the new system. The new system has obvious potential to make it easier to obtain repeated measurements in order to assess progress of the disease or response of treatment.

\section{ACKNOWLEDGEMENTS}

The authors would like to acknowledge the contributions of Mr ML Newey, Mr CJ Kershaw, Mrs A Clarke, Mrs P Rouse and Mrs P Redwood who all took part in carrying out the study. Mrs Hanna Heikkinen MSc contributed to the statistical part of the study.

\section{REFERENCES}

[1] Atroshi, I., Gummeson, C., Johnsson, R., Ornstein, E., Ranstam, J. and Rosen, I. (1999) Prevalence of carpal tunnel syndrome in a general population. Journal of the American Medical Association, 282, 153-158.
[2] Bland, J.D.P. and Rudolfer, S.M. (2003) Clinical surveillance of carpal tunnel syndrome in two areas of the United Kingdom, 1991-2001. Journal of Neurol Neurosurg Psychiatry, 74, 1674-1679.

doi:10.1136/jnnp.74.12.1674

[3] American Academy of Neurology and the American Academy of Physical Medicine and Rehabilitation. Practice parameter (2002) Electrodiagnostic studies in carpal tunnel syndrome. Report of the American Association of Electrodiagnostic Medicine. Neurology, 58, 1589-1592.

[4] Padua, L., LoMonaco, M., Gregori, B., Valente, E.M., Padua, R. and Tonali, P. (1997) Neurophysiological classification and sensitivity in 500 carpal tunnel syndrome hands. Acta Neurologica Scandinavica, 96, 211-217. doi:10.1111/j.1600-0404.1997.tb00271.x

[5] Department of Health (2007) Transforming Clinical Neurophysiology Diagnostic Services to deliver 18 Weeks. A Good Practice Guide.

[6] Uncini, A., Di Muzio, A., Awad, J., Manente, G., Tafuro, M. and Gambi, D. (1993) Sensitivity of three medianto-ulnar comparative tests in diagnosis of mild carpal tunnel syndrome. Muscle Nerve, 16, 1366-1373. doi:10.1002/mus.880161215

[7] Tolonen, U., Kallio, M., Ryhänen, J., Raatikainen, T., Honkala, V. and Lesonen, V. (2007) A handheld nerve conduction measuring device in carpal tunnel syndrome. Acta Neurologica Scandinavica, 115, 390-397.

[8] Levine, D.W., Simmons, B.P., Koris, M.J., et al. (1993) A self-administered questionnaire for the assessment of severity of symptoms and functional status in carpal tunnel syndrome. Journal of Bone and Joint Surgery, 75, 15851592.

[9] Bland, J.M. and Altman, D.G. (1986) Statistical methods for assessing agreement between two methods of clinical measurement. Lancet, 1, 307-310.

[10] Hobby, J.L. and Dias, J.J. (2006) A review of hand surgery provision in England. Journal of Hand Surgery, 2, 230-235.

[11] Repaci, M., Torrieri, F., Di Blasio, F. and Uncini, A. (1999) Exclusive electrophysiological motor involvement in carpal tunnel syndrome. Clinical Neurophysiology, 110, 1471-1474. doi:10.1016/S1388-2457(99)00071-1

[12] Chang, M.H., Liu, L.H., Lee, Y.C., Wei, S.J., Chiang, H.L. and Hsieh, P.F. (2006) Comparison of sensitivity of transcarpal median motor conduction velocity and conventional conduction techniques in electro diagnosis of carpal tunnel syndrome. Clinical Neurophysiology, 117, 984-991. doi:10.1016/j.clinph.2006.01.015

[13] Eisen, A., Schulzer, M., Pant, B., MacNeil, M., Stewart, H., Trueman, S. and Mak, E. (1993) Receiver operating characteristic curve analysis in the prediction of carpal tunnel syndrome: A model for reporting electrophysiological data. Muscle Nerve, 16, 787-796. doi:10.1002/mus. 880160715 